\title{
Análisis del cambio en la conectividad estructural del paisaje (1975-2008) de la cuenca del lago Cuitzeo, Michoacán, México ${ }^{1}$
}

\author{
Camilo Andrés Correa Ayram², Manuel E. Mendoza 3 y Erna López Granados ${ }^{4}$
}

\begin{abstract}
RESUMEN
La cuenca del lago Cuitzeo presenta tipos de cobertura arbórea y arbustiva de alta importancia ecológica que a través del tiempo han sido fragmentadas a causa de la presión antrópica. Este estudio provee un análisis del cambio en la conectividad estructural de la cuenca del lago Cuitzeo, Michoacán entre 1975 y 2008. Se utilizaron mapas de cobertura de los años 1975, 1996, 2000, 2003 y 2008. En cada año de estudio se aplicaron métricas de composición, configuración, aislamiento y conectividad en el nivel de clase y se llevó a cabo el análisis comparativo. Las coberturas de tipo arbóreo y arbustivo presentaron una conectividad estructural baja en contraste con las coberturas de origen antrópico las cuales dominaron el paisaje a través del tiempo. Se discuten los cambios en la conectividad estructural y la fragmentación y sus implicaciones para el manejo del paisaje y la conservación de la biodiversidad en esta región.
\end{abstract}

Palabras clave: Métricas del paisaje, fragmentación, cambio del paisaje, cobertura arbórea y arbustiva.

\begin{abstract}
The Cuitzeo Lake Basin is covered with forests and scrublands with high ecological importance, which have experienced fragmentation resulting from human activities. In this study we analyzed changes in the structural connectivity of the Cuitzeo Lake basin, Michoacan, between 1975 and 2008. We used land cover databases for the years 1975, 1996, 2000, 2003 and 2008. For each year, landscape composition metrics were applied, such as isolation and structural connectivity metrics at the landscape and class levels. Finally we performed a comparative analysis. The forest cover presented low structural connectivity values in contrast to the human activity related land covers, which dominated the landscape over time. The information on changes in landscape structural connectivity and fragmentation from this study provide a useful tool for landscape management and biodiversity conservation.
\end{abstract}

Key words: Landscape metrics, fragmentation, landscape change, forest cover and scrubland.

\footnotetext{
1 Los autores agradecen al proyecto "Dinámica espacio temporal de los fragmentos de bosque mesófilo de montaña del Sistema Volcánico Transversal del estado de Michoacán bajo un enfoque de paisaje", financiado por DGAPA-PAPIIT (IN1118119). El autor principal agradece a CONACYT por la beca No. 256412 otorgada para realizar los estudios de maestría en Geografía de la UNAM. Artículo recibido el 26 de abril de 2013, aceptado el 13 de enero de 2014 y corregido el 27 de enero de 2014.
}

\footnotetext{
2 Centro Investigaciones en Geografía Ambiental, Universidad Nacional Autónoma de México (México).E-mail: correa.camilo@gmail.com

3 Centro Investigaciones en Geografía Ambiental, Universidad Nacional Autónoma de México (México). E-mail: mmendoza@ciga.unam.mx

4 Departamento de Geología y Mineralogía, Instituto de Investigaciones en Ciencias de la Tierra, Universidad Michoacana de San Nicolás de Hidalgo (México). E-mail: ernalopez2004@yahoo.com.mx
} 
La conectividad del paisaje es necesaria para sostener y mantener la estabilidad de los procesos ecológicos que se encuentran espacialmente relacionados entre sí, como la dispersión, el flujo genético entre poblaciones aisladas, la migración y a largo plazo la evolución de las especies. En este sentido, el mantenimiento de la conectividad se convierte en una condición clave para la persistencia de la biodiversidad y requisito ecológico indispensable en las prácticas adecuadas de conservación y planificación del territorio (Laita et al., 2011).

Taylor et al. (2006) consideran dos tipos de conectividad: estructural y funcional. La conectividad estructural o espacial se refiere a las relaciones de continuidad y adyacencia entre los fragmentos de un tipo de cobertura, mientras que la conectividad funcional se refiere a la continuidad de los flujos ecológicos que se dan a través del paisaje (Taylor et al., 2006). La conectividad estructural aumenta cuando los fragmentos de un tipo de cobertura están más cercanos entre sí. En este sentido, la conectividad estructural es analizada mediante la aplicación de índices o métricas del paisaje calculadas por medio de un sistema de información geográfica (Gustafson, 1998). Las métricas del paisaje proveen información útil acerca de las relaciones espaciales en el paisaje (O’Neill et al., 1988). Como por ejemplo, la proximidad, la agregación y el aislamiento entre fragmentos que conforman un tipo de cobertura en particular (Botequilha et al., 2006). De esta manera, el análisis de la conectividad estructural a través del tiempo es relevante porque permite describir los patrones de cambio en la configuración espacial de los elementos estructurales del paisaje (matriz, fragmento y corredor) generando información clave acerca del nivel de intervención antrópica en un momento y área determinada (Forman, 1995), siendo un componente fundamental en el monitoreo de la integridad ecológica para la conservación de la biodiversidad.

Li y Jianguo (2004) reportan algunas limitaciones sobre el uso de las métricas en el estudio de la transformación del paisaje, como la deficiencia de los índices para relacionar cambios estructurales con cambios cualitativos y la insensibilidad para asociarse con los atributos funcionales del paisaje. Asi- mismo, Saura y Martínez-Millán (2001) señalan que la extensión espacial y el nivel de detalle sobre el cual se calculan las métricas del paisaje influyen en los resultados de estos análisis, generando incertidumbre en cuanto a la solidez y la fiabilidad de los resultados y limita la comparabilidad de la estructura del paisaje a través del tiempo. Sin embargo, las ventajas de su aplicación han sido sólidamente ejemplificadas por Gustafson (1998), el cual señala que el potencial del uso de las métricas radica en su utilidad para el análisis de la configuración del paisaje, por ejemplo, comparando paisajes cartografiados de la misma manera, evaluando el mismo paisaje en diferentes años, o comparando el mismo paisaje bajo escenarios alternativos (Botequilha et al., 2006).

En el caso de la cuenca del lago Cuitzeo, el cambio de la conectividad estructural no se ha evaluado. Sin embargo, existe una amplia disponibilidad de bases de datos generadas en estudios previos sobre cambio de cobertura y uso del suelo las cuales fueron utilizadas para desarrollar el análisis de cambio (López et al., 2006; Mendoza et al., 2011). También, el acelerado aumento de la población urbana de la cuenca, la cual presentó un incremento del $187 \%$ en los últimos 30 años (INEGI, 1970; INEGI, 2010), ha generado una fuerte presión sobre los recursos naturales de la misma, los procesos de transformación antrópica han configurado un paisaje dominado por coberturas y usos del suelo como los pastizales y cultivos que han remplazado las coberturas naturales de tipo arbóreo y arbustivo, generando problemas ambientales como la fragmentación del hábitat y la reducción de la conectividad ecológica.

Este trabajo, enfocado en el estudio de conectividad estructural, es prerrequisito para un posterior análisis de conectividad funcional en la cuenca, en el cual se utilizaron para su modelamiento, datos de aptitud de hábitat y de dispersión de dos mamíferos terrestres (Linx rufus y Bassariscus astutus) con características ecológicas contrastantes (Correa et al., 2014).

El presente estudio tiene como propósito conocer el cambio en la estructura y conectividad del paisaje en la cuenca del lago Cuit- 
zeo y realizar su monitoreo retrospectivo. En este sentido, este estudio constituye un aporte al conocimiento de la ecología del paisaje de un área ecológica importante que históricamente ha presentado una fuerte presión sobre sus recursos naturales.

\section{Materiales y métodos}

La cuenca del lago Cuitzeo se encuentra ubicada entre los $19^{\circ} 30^{\prime}$ y $20^{\circ} 05^{\prime}$ latitud norte y $100^{\circ} 35^{\prime}$ y $101^{\circ} 30^{\prime}$ longitud oeste en los límites del estado de Michoacán y Guanajuato (Figura $N^{0} 1$ ). Se localiza en el Sistema Volcánico Transversal y presenta aproximadamente una superficie de $4.000 \mathrm{~km}^{2}$ (Mendoza et al., 2011). La cuenca presenta una altitud mínima de 1.830 msnm y una máxima de $3.420 \mathrm{msnm}$. Asimismo, la cuenca es de tipo endorreico en donde el drenaje superficial se concentra en el área más baja de la misma formando el lago Cuitzeo (Morales, 2010). La temperatura media anual es aproximadamente de $16^{\circ}$ a $18^{\circ} \mathrm{C}$ para casi toda la cuenca, sin embargo en las partes altas se presentan temperaturas medias entre 14 a $16^{\circ} \mathrm{C}$. La pre- cipitación promedio anual es menor a 1.000 mm (Carlón y Mendoza, 2007; Vidal- Zepeda, 2010).

La cuenca del lago Cuitzeo está conformada por lomeríos y colinas constituidas por materiales volcánicos del Terciario superior al Plioceno, sobre los cuales se han desarrollado vertisoles, luvisoles, andosoles, acrisoles y feozems (Mendoza et al., 2001). Según el mapa de cobertura y uso del suelo al año 2008, las coberturas de origen antrópico como los pastizales, cultivos de temporal y riego, así como las plantaciones forestales dominan el $50 \%$ de la superficie de la cuenca, indicando el alto grado de intervención antrópica de la misma (Figura $\mathrm{N}^{\circ}$ 2). Le siguen las coberturas de tipo arbóreo y arbustivo como los bosques con un 19\% y los matorrales con un 13\% (Cuadro $N^{\circ} 1$ ). Los asentamientos humanos ocupan el 5\% del total de la cuenca (Mendoza et al., 2011). La cuenca de Cuitzeo abarca 28 municipios de los cuales 5 pertenecen al estado de Guanajuato y 23 al estado de Michoacán. En total incluye 392 asentamientos humanos). La

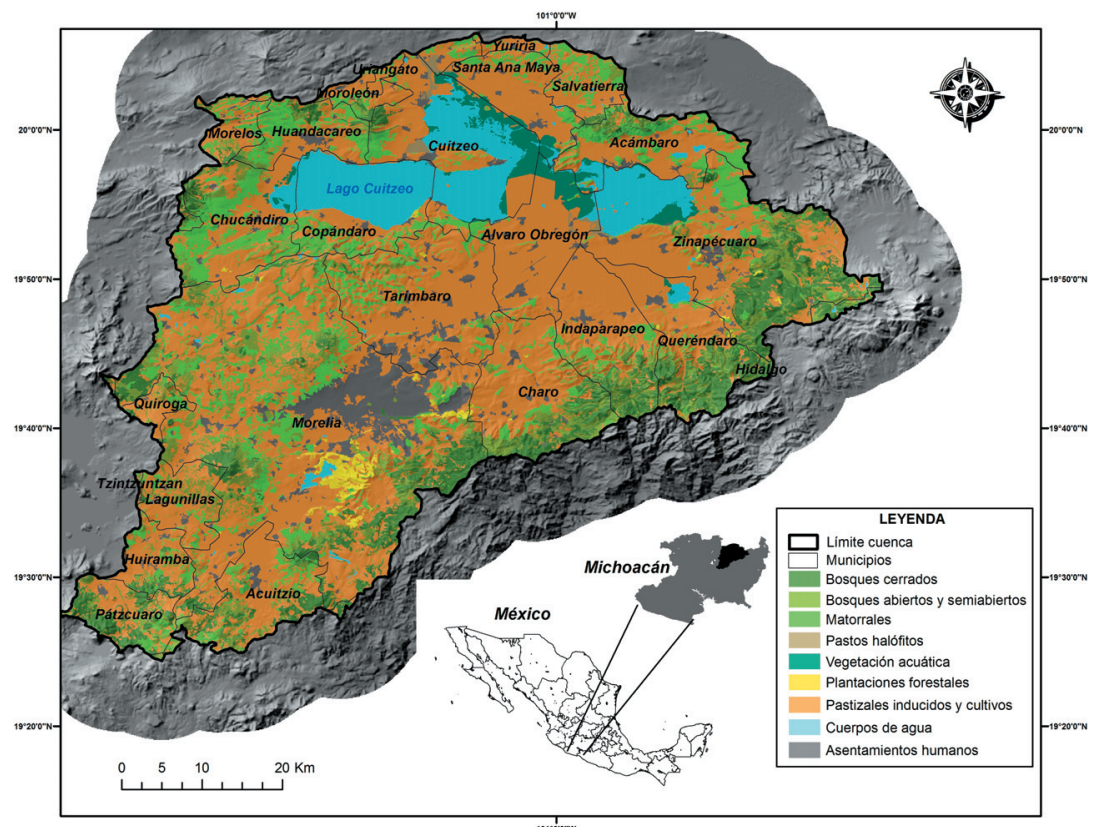

Fuente: Elaboración propia. 
Cuadro $\mathrm{N}^{\circ} 1$

Tipos de cobertura y uso del suelo

\begin{tabular}{|c|c|}
\hline Tipo de cobertura & Descripción \\
\hline Bosques cerrados & $\begin{array}{l}\text { Asociaciones arbóreas de coníferas (pino, oyamel, cedro blanco) } \\
\text { bosques de encino y bosques mixtos. El dosel ocupa más del } \\
90 \% \text { de su superficie. }\end{array}$ \\
\hline $\begin{array}{l}\text { Bosques abiertos y } \\
\text { semiabiertos }\end{array}$ & $\begin{array}{l}\text { Asociaciones arbóreas de coníferas (pino, oyamel, cedro blanco) } \\
\text { bosques de encino y bosques mixtos. El dosel ocupa entre el } 60 \% \text { - } \\
90 \% \text { de su superficie. }\end{array}$ \\
\hline Matorrales & $\begin{array}{l}\text { Asociación vegetal dominada por arbustos. En general correspon- } \\
\text { de a matorrales secundarios de bosque tropical caducifolio. El } \\
\text { dosel ocupa entre el } 60 \%-90 \% \text { de su superficie. }\end{array}$ \\
\hline Pastos halófitos & $\begin{array}{l}\text { Vegetación herbácea entre } 10 \mathrm{~cm}-60 \mathrm{~cm} \text { de altura aproximada- } \\
\text { mente, asociada a zonas de desecación del lago. }\end{array}$ \\
\hline Vegetación acuática & Los tipos más comunes corresponden al tular y al carrizal. \\
\hline Plantaciones forestales & $\begin{array}{l}\text { Superficies arboladas en su mayoría por especies exóticas como } \\
\text { el eucalipto. }\end{array}$ \\
\hline $\begin{array}{l}\text { Pastizales inducidos y } \\
\text { cultivos }\end{array}$ & $\begin{array}{l}\text { Vegetación herbácea dominante mezclada con áreas ocupadas } \\
\text { por frutales, cultivos de secano y cultivos con riego artificial. }\end{array}$ \\
\hline Cuerpos de agua & $\begin{array}{l}\text { Masas de agua de origen natural como el lago Cuitzeo y artificia- } \\
\text { les como presas y reservorios. }\end{array}$ \\
\hline Asentamientos humanos & Áreas residenciales agregadas, comerciales, servicios y carreteras. \\
\hline
\end{tabular}

Fuente: Elaboración propia.

población total de la cuenca en el año 1970 correspondía a 380,782 habitantes creciendo a 944,606 habitantes al año 2010 (INEGI, 1970, 2010).

\section{Preparación de los datos}

Los datos geográficos de cobertura y uso del suelo existentes para la cuenca fueron integrados y analizados para los distintos tiempos (1975, 1996, 2000, 2003 y 2008), dichos datos presentan diferencias en sus características espaciales y espectrales, por esta razón, para el análisis comparativo fue necesario unificarlos en cuanto a su leyenda y escala de análisis (1:80.000). Inicialmente las bases de datos fueron generalizadas de 19 a 9 tipos de cobertura (Cuadro $N^{\circ} 1$; Figura $N^{\circ} 2$ ). La interpretación de los años 1975 y 2000 se realizó sobre fotografías aéreas convencionales pancromáticas $\mathrm{B} / \mathrm{N}$ a escala 1:50.000 y 1: 37.000 respectivamente. La base de datos del año 2000 sirvió de referencia para interpretar los años 1996, 2003 y 2008. Esta base de datos es considerada de referencia porque su nivel de confianza es superior al 90\%, el error de ubicación de los puntos de control se fijó en 0,4 mm (o mejor) en el mapa base a 1:50.000 (López et al., 2006). Para la interpretación del año 1996 se utilizaron ortofotos pancromáticas ( $2 \times 2 \mathrm{~m})$, para el 2003 fue usada una imagen Landsat ETM+ fusionada con la banda pancromática (15 x 15 m) (Mendoza et al., 2011) y el año 2008 fue interpretado sobre una imagen SPOT fusionada a 2,5 m por pixel (Mendoza et al., 2010). El área mínima cartografiable (AMC) utilizada en esta investigación fue de 
3 ha, valor cercana al AMC más pequeña aceptable, que corresponde a $2 \times 2 \mathrm{~mm}$ (Priego et al., 2010). El AMC se estandarizó para todos las años interpretados. Todas las bases de datos espaciales se encuentran en proyección UTM (Universal Transversa de Mercator), datum NAD 27 (North American Datum 1927), zona 14 norte.

Figura $\mathrm{N}^{\circ} 2$

Distribución de los tipos de cobertura y uso del suelo en la cuenca en 1975, 1996, 2000, 2003 y 2008 y su representatividad en el paisaje
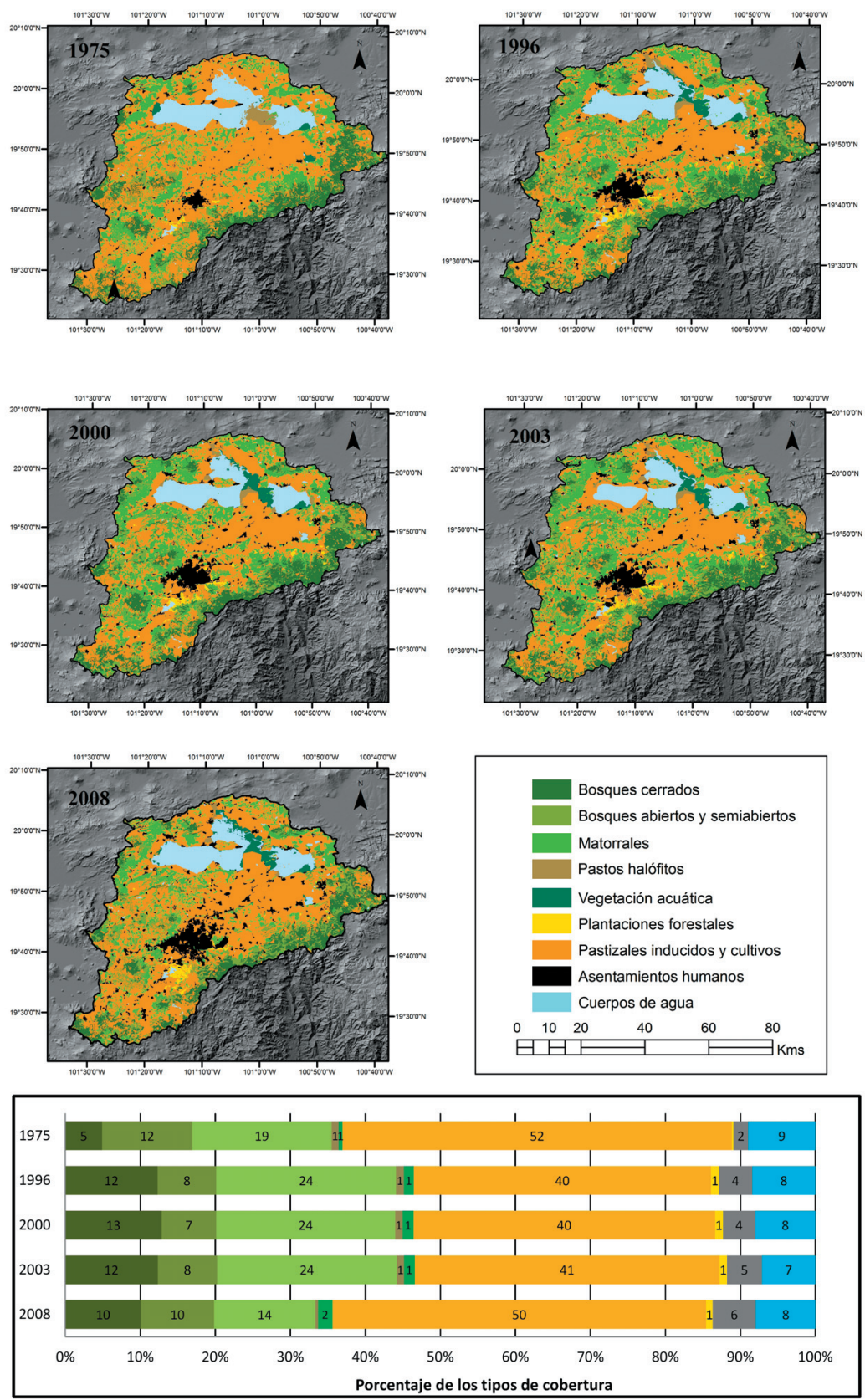

Fuente: Elaboración propia. 


\section{Aplicación de métricas del paisaje}

La estructura del paisaje puede ser analizada en tres niveles, dependiendo de la finalidad del estudio: nivel de fragmento, nivel de clase y nivel de paisaje (Gustafson, 1998; McGarigal et al., 2002). En este caso, para describir los cambios estructurales en la conectividad y la fragmentación del paisaje el nivel adecuado de análisis corresponde al de clase.

Se utilizó el término "fragmento" como sinónimo de "parche" (Sarmiento, 2001), refiriéndose a los fragmentos como las unidades morfológicas relativamente homogéneas que se pueden diferenciar en el territorio (Forman, 1995), sin discriminar si el origen del fragmento del tipo de cobertura es natural o antrópico.

En el nivel de clase, una clase corresponde a un tipo de cobertura. La mayoría de las métricas en este nivel pueden ser interpretadas como índices de fragmentación porque miden la configuración espacial de un tipo de cobertura en particular (Botequilha et al., 2006).
Con el fin de cuantificar la estructura y conectividad del paisaje en cada periodo de tiempo se calcularon originalmente un total de diez índices de paisaje. Sin embargo, se aplicó un análisis de correlación entre cada par de índices para descartar la generación de información redundante (Ritters et al., 1995; Hargis et al., 1998; Neel et al., 2004;). Finalmente, se utilizaron siete índices de paisaje (Cuadro $\mathrm{N}^{\circ} 2$ ) correspondientes a: métricas básicas de área y configuración de los fragmentos: porcentaje de cobertura (PLAND), tamaño promedio del fragmento (MPS), índice del fragmento más grande (LPI) y densidad de fragmentos (PD); b: métricas de conectividad y aislamiento: Índice de proximidad (MPI)). Conectancia (CONNECT), Cohesión (COHESION).

Estos índices fueron calculados por medio del programa FRAGSTATS 3.3 (McGarigal et al., 2002) el cual corresponde al programa de cómputo más popular para el cálculo de métricas del paisaje en formato raster (Zaragozí et al., 2012), posteriormente se llevó a cabo el análisis comparativo entre las fechas de la cartografía de cobertura y uso del suelo (1976, 1996, 2000, 2003 у 2008).

Cuadro $\mathrm{N}^{\circ} 2$

Índices del paisaje seleccionados para el estudio.

\begin{tabular}{|c|c|c|c|}
\hline Índice & Acrónimo & Descripción & Referencias \\
\hline $\begin{array}{l}\text { Porcentaje del } \\
\text { Paisaje }\end{array}$ & PLAND & $\begin{array}{l}\text { Porcentaje ocupado por un tipo de cobertura en } \\
\text { el total del paisaje. Es la métrica más elemental en } \\
\text { el estudio de los patrones de paisaje. Los cambios } \\
\text { del porcentaje en el tiempo dan información acer- } \\
\text { ca del incremento y el decrecimiento de las áreas } \\
\text { de cierto tipo de cobertura. PLAND se aproxima a } \\
0 \text { cuando el tipo de cobertura disminuye su área y } \\
\text { se acerca a } 100 \text { cuando domina el total del paisa- } \\
\text { je. Unidad: porcentaje. }\end{array}$ & Turner, 1990 \\
\hline $\begin{array}{l}\text { Tamaño } \\
\text { Promedio del } \\
\text { Fragmento }\end{array}$ & MPS & $\begin{array}{l}\text { El tamaño promedio del fragmento (MPS) es el } \\
\text { promedio aritmético del tamaño de cada fragmen- } \\
\text { to de cierto tipo de cobertura, este índice mide } \\
\text { qué tan dividido se encuentra un tipo de cober- } \\
\text { tura. Si el fragmento ocupa el total del paisaje, } \\
\text { el área del paisaje será el valor máximo del MPS. } \\
\text { Unidad: hectáreas. }\end{array}$ & $\begin{array}{l}\text { Forman \& Go- } \\
\text { dron, 1981; } \\
\text { Gergel \& Tur- } \\
\text { ner, } 2002\end{array}$ \\
\hline
\end{tabular}




\begin{tabular}{|c|c|c|c|}
\hline Índice & Acrónimo & Descripción & Referencias \\
\hline $\begin{array}{l}\text { Índice del } \\
\text { Fragmento } \\
\text { más Grande }\end{array}$ & LPI & $\begin{array}{l}\text { Porcentaje que ocupa el fragmento más grande en } \\
\text { el total del tipo de coberturas si se calcula en el ni- } \\
\text { vel de clase. LPI se aproxima a } 0 \text { cuando el área de } \\
\text { fragmento más grande de la clase correspondiente } \\
\text { es muy pequeña y es igual a } 100 \text { cuando el total del } \\
\text { paisaje consiste de un solo fragmento que ocupa el } \\
100 \% \text { del área del mismo. Unidad: porcentaje. }\end{array}$ & $\begin{array}{l}\text { Forman, 1995; } \\
\text { With \& King, } \\
\text { 1999; Saura \& } \\
\text { Martínez-Mi- } \\
\text { llán, } 2001\end{array}$ \\
\hline $\begin{array}{l}\text { Densidad de } \\
\text { Fragmentos }\end{array}$ & PD & $\begin{array}{l}\text { Número de fragmentos de un tipo de cobertura } \\
\text { en } 100 \text { hectáreas. El valor mínimo de NP ocurre } \\
\text { cuando el total del paisaje es dominado por un } \\
\text { solo fragmento que ocupa toda el área del mismo. } \\
\text { Unidad: Ninguna. }\end{array}$ & $\begin{array}{l}\text { Franklin \& For- } \\
\text { man, } 1987 ; \\
\text { Saura \& Mar- } \\
\text { tínez-Millán, } \\
2001\end{array}$ \\
\hline $\begin{array}{l}\text { Índice de } \\
\text { proximidad } \\
\text { media }\end{array}$ & MPI & $\begin{array}{l}\text { Caracteriza el grado de aislamiento espacial de los } \\
\text { fragmentos, tomando en cuenta todos los fragmen- } \\
\text { tos más próximos que se encuentran dentro de un } \\
\text { radio de búsqueda especificado. Rango }>0 \text {, sin } \\
\text { límites; Altos valores de proximidad indican que } \\
\text { fragmentos vecinos, del mismo tipo de cobertura, } \\
\text { están menos aislados, grandes y agregados. Bajos } \\
\text { valores indican que los fragmentos se encuentran } \\
\text { aislados y pueden tener tamaños pequeños. Uni- } \\
\text { dad: Ninguna }\end{array}$ & $\begin{array}{l}\text { Gustafson \& } \\
\text { Parker, 1994; } \\
\text { Hargis et al., } \\
1998\end{array}$ \\
\hline Conectancia & CONNECT & $\begin{array}{l}\text { Mide el grado de conexión física entre los frag- } \\
\text { mentos que conforman un paisaje, siendo un } \\
\text { indicador directo de conectividad espacial. Es de- } \\
\text { finido por el número de enlaces funcionales entre } \\
\text { fragmentos del mismo tipo donde cada fragmento } \\
\text { está conectado o no, basado en un criterio de } \\
\text { distancia. Rango de } 0 \text { a } 100 \text {; CONNECT es igual } \\
\text { a } 0 \text { cuando la clase consiste de un solo fragmento } \\
\text { o ninguno de los fragmentos está conectado, y es } \\
\text { igual a } 100 \text { cuando todos los fragmentos de la cla- } \\
\text { se están conectados. Unidad: porcentaje. }\end{array}$ & $\begin{array}{l}\text { McG arigal } \\
\text { et al., 2002; } \\
\text { Kindlmann y } \\
\text { Burel, 2008; }\end{array}$ \\
\hline $\begin{array}{l}\text { Índice de } \\
\text { Cohesión }\end{array}$ & COHESIÓN & $\begin{array}{l}\text { Mide el grado de agregación y la dominancia de } \\
\text { las coberturas que conforman un paisaje deter- } \\
\text { minado. En este sentido, es un indicador de la } \\
\text { conectividad física de un paisaje o de un tipo de } \\
\text { cobertura. Rango de } 0 \text { a } 100 \text {; el valor de COHE- } \\
\text { SION se incrementa a medida que la agregación y } \\
\text { la agrupación de las coberturas aumentan. }\end{array}$ & $\begin{array}{l}\text { Schumaker, } \\
\text { 1996; Gustaf- } \\
\text { son, } 1998\end{array}$ \\
\hline
\end{tabular}

Fuente: Modificado de McGarigal et al., 2002 y Botequilha et al., 2006.

\section{Resultados}

\section{Configuración espacial y composición en} el nivel de clase

Se describen los resultados de configuración y composición del paisaje en el nivel de clase, considerando las métricas de tamaño promedio de fragmentos (MPS), densidad de fragmentos (PD), índice del fragmento más grande (LPI) y porcentaje de cobertura (PLAND). En general el comportamiento entre el MPS y PD difiere sustancialmente (Cuadro $N^{\circ} 3$ ). Los matorrales registran una alta den- 
sidad entre 1975 y 2003 y tamaños de fragmentos relativamente pequeños; sin embargo, el valor decrece considerablemente en el año 2008. En cuanto al índice de MPS, valores bajos se asocian a la clase matorrales, los cuales prácticamente presentan un comportamiento estable a través del tiempo.

Cuadro $\mathrm{N}^{\circ} 3$

Cambios en los índices del paisaje en el nivel de clase

\begin{tabular}{|c|c|c|c|c|c|c|c|}
\hline CLASE & PLAND & MPS & LPI & PD & MPI & CONNECT & COHESION \\
\hline \multicolumn{8}{|l|}{1975} \\
\hline Bosques cerrados & 4.90 & 17,31 & 0,25 & 0,28 & 170,48 & 0,48 & 97,39 \\
\hline $\begin{array}{l}\text { Bosques abiertos y } \\
\text { Semiabiertos }\end{array}$ & 12,02 & 111,64 & 6,67 & 0,11 & $38.667,51$ & 1,17 & 99,78 \\
\hline Matorrales & 18,58 & 48,85 & 1,12 & 0,38 & $1,422,03$ & 0,40 & 99,06 \\
\hline Pastos halófitos & 0,96 & 167,34 & 0,88 & 0,01 & 79,03 & 8,70 & 99,50 \\
\hline Vegetación acuática & 0,50 & 143,59 & 0,17 & 0,00 & 486,11 & 7,69 & 99,00 \\
\hline $\begin{array}{l}\text { Plantaciones } \\
\text { forestales }\end{array}$ & 0,22 & 37,34 & 0,08 & 0,01 & 104,22 & 4,35 & 98,03 \\
\hline $\begin{array}{l}\text { Pastizales inducidos } \\
\text { y cultivos }\end{array}$ & 51,87 & 185,24 & 45,68 & 0,28 & $409.747,74$ & 0,54 & 99,97 \\
\hline Cuerpos de agua & 8,93 & 606,24 & 8,64 & 0,01 & $25.793,15$ & 1,34 & 99,89 \\
\hline $\begin{array}{l}\text { Asentamientos } \\
\text { humanos }\end{array}$ & 2,00 & 19,67 & 0,56 & 0,10 & 172,42 & 0,36 & 97,54 \\
\hline \multicolumn{8}{|l|}{1996} \\
\hline Bosques cerrados & 12,29 & 102,51 & 2,25 & 0,12 & $5.920,30$ & 0,80 & 99,51 \\
\hline $\begin{array}{l}\text { Bosques abiertos y } \\
\text { semiabiertos }\end{array}$ & 7,84 & 24,24 & 0,43 & 0,32 & 287,23 & 0,46 & 97,99 \\
\hline Matorrales & 23,96 & 61,88 & 4,61 & 0,39 & $10.401,78$ & 0,35 & 99,54 \\
\hline Pastos halófitos & 1,04 & 99,50 & 0,45 & 0,01 & $1.314,27$ & 4,88 & 99,07 \\
\hline Vegetación acuática & 1,34 & 233,10 & 0,99 & 0,01 & $10.474,17$ & 7,91 & 99,57 \\
\hline $\begin{array}{l}\text { Plantaciones } \\
\text { forestales }\end{array}$ & 1,04 & 39,75 & 0,27 & 0,03 & 774,11 & 1,74 & 98,53 \\
\hline $\begin{array}{l}\text { Pastizales inducidos } \\
\text { y cultivos }\end{array}$ & 39,62 & 125,28 & 18,36 & 0,32 & $43.986,15$ & 0,43 & 99,83 \\
\hline Cuerpos de agua & 8,41 & 410,65 & 5,99 & 0,02 & $27.803,58$ & 1,29 & 99,79 \\
\hline $\begin{array}{l}\text { Asentamientos } \\
\text { humanos }\end{array}$ & 4,45 & 39,96 & 1,77 & 0,11 & 383,41 & 0,40 & 98,77 \\
\hline \multicolumn{8}{|l|}{2000} \\
\hline Bosques cerrados & 12,85 & 107,83 & 2,38 & 0,12 & $6.967,38$ & 0,81 & 99,49 \\
\hline $\begin{array}{l}\text { Bosques abiertos y } \\
\text { semiabiertos }\end{array}$ & 7,29 & 25,23 & 0,45 & 0,29 & 252.77 & 0,46 & 97,94 \\
\hline Matorrales & 23,85 & 70,77 & 2,76 & 0,34 & $6.212,23$ & 0,37 & 99,53 \\
\hline Pastos halófitos & 0,99 & 113,28 & 0,28 & 0,01 & 205,76 & 3,53 & 98,78 \\
\hline Vegetación acuática & 1,45 & 304,65 & 1,05 & 0,00 & 381,13 & 7,02 & 99,48 \\
\hline
\end{tabular}


Continuación Cuadro $N^{\circ} 3$

\begin{tabular}{|c|c|c|c|c|c|c|c|}
\hline CLASE & PLAND & MPS & LPI & PD & MPI & CONNECT & COHESION \\
\hline $\begin{array}{l}\text { Plantaciones } \\
\text { forestales }\end{array}$ & 1,05 & 45,67 & 0,28 & 0,02 & 544,33 & 1,72 & 98,60 \\
\hline $\begin{array}{l}\text { Pastizales inducidos } \\
\text { y cultivos }\end{array}$ & 40,20 & 142,66 & 19,01 & 0,28 & $41.676,17$ & 0,46 & 99,84 \\
\hline Cuerpos de agua & 8,00 & 485,10 & 5,75 & 0,02 & $13.522,05$ & 0,93 & 99,81 \\
\hline $\begin{array}{l}\text { Asentamientos } \\
\text { humanos }\end{array}$ & 4,34 & 45,94 & 1,86 & 0,09 & 950,95 & 0,39 & 98,85 \\
\hline \multicolumn{8}{|l|}{2003} \\
\hline Bosques cerrados & 12,32 & 97,87 & 2,28 & 0,13 & $6.946,81$ & 0,78 & 99,51 \\
\hline $\begin{array}{l}\text { Bosques abiertos y } \\
\text { semiabiertos }\end{array}$ & 7,91 & 23,21 & 0,44 & 0,34 & 246,93 & 0,45 & 97,94 \\
\hline Matorrales & 23,95 & 60,76 & 2,29 & 0,39 & $4.863,02$ & 0,35 & 99,46 \\
\hline Pastos halófitos & 0,98 & 100,15 & 0,28 & 0,01 & 741,22 & 3,51 & 98,78 \\
\hline Vegetación acuática & 1,41 & 297,98 & 0,99 & 0,00 & 334,50 & 9,94 & 99,47 \\
\hline $\begin{array}{l}\text { Plantaciones } \\
\text { forestales }\end{array}$ & 0,99 & 37,39 & 0,17 & 0,03 & 396,94 & 1,62 & 98,25 \\
\hline $\begin{array}{l}\text { Pastizales inducidos } \\
\text { y cultivos }\end{array}$ & 40,65 & 127,14 & 18,63 & 0,32 & $37.963,94$ & 0,43 & 99,84 \\
\hline Cuerpos de agua & 7,11 & 418,70 & 4,75 & 0,02 & $3.588,19$ & 0,97 & 99,76 \\
\hline $\begin{array}{l}\text { Asentamientos } \\
\text { humanos }\end{array}$ & 4,67 & 40,57 & 1,82 & 0,12 & 841,34 & 0,40 & 98,84 \\
\hline \multicolumn{8}{|l|}{2008} \\
\hline Bosques cerrados & 10,08 & 101,63 & 1,33 & 0,10 & $2.784,70$ & 0,88 & 99,30 \\
\hline $\begin{array}{l}\text { Bosques abiertos y } \\
\text { semiabiertos }\end{array}$ & 9,74 & 38,49 & 0,55 & 0,25 & 506,85 & 0,54 & 98,52 \\
\hline Matorrales & 13,53 & 53,06 & 1,21 & 0,26 & $1.171,52$ & 0,45 & 98,98 \\
\hline Pastos halófitos & 0,34 & 89,89 & 0,14 & 0,00 & 1,62 & 4,76 & 98,71 \\
\hline Vegetación acuática & 1,94 & 88,07 & 1,38 & 0,02 & $3.175,77$ & 4,78 & 99,51 \\
\hline $\begin{array}{l}\text { Plantaciones } \\
\text { forestales }\end{array}$ & 0,87 & 52,20 & 0,50 & 0,02 & 597,77 & 3,89 & 99,09 \\
\hline $\begin{array}{l}\text { Pastizales inducidos } \\
\text { y cultivos }\end{array}$ & 49,81 & 174,01 & 40,85 & 0,29 & $249.316,03$ & 0,50 & 99,96 \\
\hline Cuerpos de agua & 7,92 & 453,13 & 5,54 & 0,02 & $4.217,55$ & 0,95 & 99,82 \\
\hline $\begin{array}{l}\text { Asentamientos } \\
\text { humanos }\end{array}$ & 5,77 & 52,34 & 2,67 & 0,11 & $1.893,17$ & 0,40 & 99,22 \\
\hline
\end{tabular}

Fuente: Elaboración propia.

Por otro lado, los bosques abiertos y semiabiertos presentan a lo largo del tiempo valores bajos del índice de MPS; en 1975 se registró el valor más alto, sin embargo, el valor decrece considerablemente en el año 1996 y presenta un comportamiento estable hasta el año 2003 y vuelve a incrementar su valor levemente en el 2008. No obstante, para 1975 los bosques abiertos y semiabiertos presentan el valor más bajo del índice PD, luego en el año 1996 el valor del índice PD se incrementa notablemente, para el año 2003 se presenta 
el valor más alto de PD y finalmente decrece en el año 2008.

En cuanto a los bosques cerrados en general presentan valores del índice MPS relativamente bajos durante todo el periodo. En el año 1975 se registró el valor más bajo, el cual se incrementa considerablemente en 1996, estabilizándose hasta el año 2008. En lo que refiere al índice de PD, los bosques cerrados presentan valores decrecientes a través del tiempo, en 1975 registran el valor más alto, el cual desciende notablemente en 1996. Posteriormente, el valor del índice de PD se estabiliza hasta el 2003 y finalmente para el 2008 decrece sustancialmente a 0,09 fragmentos/ 100 ha correspondiendo al valor más bajo del índice de PD entre las coberturas de bosque y matorral.

Los bosques cerrados a través del tiempo presentan un leve incremento en el valor del índice LPI entre 1975 y 1996, se estabiliza hasta el año 2003 y decrece gradualmente para el 2008. En cuanto a los bosques abiertos y semiabiertos, registran valores muy bajos a través del tiempo. Sin embargo, en 1975 se presenta el valor más alto, el cual decrece considerablemente en 1996 y a través del tiempo se incrementa ligeramente hasta el 2008. Un comportamiento del índice LPI contrario al de los bosques abiertos y semiabiertos se registró en la cobertura de matorrales los cuales presentaron el valor más bajo en 1975, posteriormente ocurre un incremento 1996, que decrece gradualmente hasta 2008. En este sentido, los fragmentos más grandes de las coberturas naturales de tipo forestal presentan tamaños muy pequeños.

Los pastizales inducidos y cultivos registraron el mayor valor de índice LPI en contraste con las demás coberturas. En 1975 el índice LPI de los pastizales y cultivos registra el valor más alto, lo que representó el 45\% del área. Sin embargo, para 1996 se presenta una reducción sustancial en el índice $\mathrm{LPI}$, el índice se estabiliza hasta el 2003 y se incrementa considerablemente en 2008.

El índice calculado para analizar la composición del paisaje en el nivel de clase corresponde al porcentaje del área de cada tipo de cobertura (PLAND). Los matorrales corresponden a la cobertura de origen natural antro- pizada con mayor representatividad en el total del paisaje. En lo que se refiere a la representatividad de los bosques cerrados, registran el valor más bajo en 1975, el cual se incrementa considerablemente en el año 1996, alcanzando su registro más alto, posteriormente se estabiliza hasta el 2003 y decrece levemente su tamaño en el año 2008. Comportamiento contrario ocurre con los bosques abiertos y semiabiertos que disminuyen considerablemente entre 1975 y 1996, el valor se estabiliza hasta el 2003 y registra un leve aumento en el 2008.

Los pastizales inducidos y cultivos correspondieron a la clase con mayor representatividad en el total del paisaje y registró valores mucho más altos en comparación con las demás coberturas. En 1975 representaba el 52\% del total del paisaje. No obstante, decrece en 1996 y su valor se estabiliza hasta el 2003, finalmente en el 2008 incrementa el porcentaje de ocupación. Por otro lado, en cuanto a representatividad le siguen los matorrales dominando el $19 \%$ del total del paisaje en 1975, más adelante para 1996 se registra un leve incremento, se estabiliza hasta el 2003 y decrece considerablemente a 13,5\% en 2008.

Es pertinente resaltar el comportamiento de los asentamientos humanos, el cual presenta un incremento gradual en su tamaño, en 1975 ocupa el 0,9\%, aumenta hasta el año $1996(4,45 \%)$ y finalmente se extiende hasta el 5,8\% en el año 2008. Asimismo, otra cobertura antrópica, como son las plantaciones forestales presenta un incremento considerable en su tamaño entre 1975 y 1996, posteriormente se estabiliza hasta el año 2003 y decrece levemente al 2008. El índice LPI se incrementa notablemente entre el 2003 y 2008, esto puede estar relacionado con la disminución del índice PD en el mismo periodo de tiempo.

\section{Conectividad y aislamiento en el nivel de} clase

Se calcularon los índices de proximidad media (MPI), conectancia (CONNECT) y cohesión (COHESION). Para el cálculo de MPI se utilizó un radio de búsqueda (Cuadro $N^{\circ}$ 2) de 10.000 m que están relacionados aproximadamente con el rango de dispersión promedio para Lynx rufus (Burtn et al., 2002; 
Hansen, 2007) el cual fue utilizado para el estudio de conectividad funcional de la cuenca (Correa, 2012).

Las coberturas de tipo arbóreo y arbustivo como los bosques cerrados, abiertos y semiabiertos y matorrales presentan valores bajos del índice de MPI. Los valores del índice MPI para los bosques abiertos y semiabiertos presentan una tendencia a decrecer a través del tiempo; entre 1975 y 1996 disminuye de 38.667 a 287 respectivamente y se mantiene estable hasta el 2003 donde su valor se incrementa a 506 en el 2008. Por otro lado, los bosques cerrados presentan el registro más bajo de las coberturas de tipo arbóreo en 1975; sin embargo presentan un incremento gradual hasta el 2003 donde decrece considerablemente el 2008.

En cuanto a la cobertura de matorrales, entre 1975 y 1996 incrementa considerablemente el valor del índice de MPI y posteriormente disminuye gradualmente hasta 2008 donde presenta el menor valor del periodo analizado.

En cuanto al índice de conectancia (CONNECT; Cuadro $\mathrm{N}^{\circ} 3$ ), en general todas las coberturas presentaron valores muy bajos teniendo en cuenta que el rango está dado entre $0 \%$ a $100 \%$ (McGarigal et al., 2002). La cobertura de pastos halófitos presenta los valores más altos en comparación con las demás. Sin embargo, el valor del índice CONNECT decrece desde 1975 hasta el 2000 y se mantiene estable al 2003, finalmente aumenta en 2008. Las coberturas de tipo arbóreo y arbustivo presentan en general registros muy bajos del índice CONNECT. Los matorrales registran los valores más bajos en lo que corresponde a dichas coberturas presentando un comportamiento estable a través del tiempo y presentado un leve incremento entre 2003 y 2008. En cuanto a los bosques abiertos y semiabiertos presentan el valor más alto del índice CONNECT en 1975. No obstante, decrecen considerablemente en 1996 manteniéndose estable hasta el 2003, finalmente aumenta ligeramente en el 2008. Esta tendencia es muy parecida a la encontrada en el índice de proximidad (MPI). Por otro lado, los bosques cerrados presentan una tendencia al incremento gradual en el índice CONNECT; sin embargo, sus valores son muy bajos; en 1975 se calculó el menor valor, el cual aumentó en 1996 posteriormente se estabiliza hasta 2003, finalmente aumentó en 2008 siendo este el año con el valor de índice CONNECT más alto registrado para dicha cobertura.

En cuanto al índice de cohesión (COHESION), en general todas las coberturas tanto naturales como antrópicas presentaron valores muy altos teniendo en cuenta que este índice se reporta entre un rango de 0 a 100 (McGarigal et al., 2002). Los bosques abiertos y semiabiertos presentaron registros altos de cohesión en 1975. Sin embargo, decrecen en 1996, posteriormente el valor se estabiliza hasta el 2003 y vuelve a incrementarse levemente en 2008. Un comportamiento contrario sucedió con la cobertura de bosques cerrados, la cual presenta el valor más bajo en comparación a las demás coberturas en 1975. Más adelante, en 1996 el valor de cohesión se estabiliza hasta el 2003 y disminuye levemente en 2008. La cobertura de matorrales en general también presenta valores altos de cohesión. Para 1975 presenta un valor de cohesión de 99; posteriormente, en 1996 el registro se incrementa y decrece levemente en el 2003, finalmente el valor más bajo del índice de cohesión se encontró en el 2008. Vale la pena resaltar la tendencia a través del tiempo de los asentamientos humanos en el que el índice de cohesión se incrementa a través del tiempo. En 1975 presentó un valor de 97,5, en 1996 aumentó a 98,7 y se estabilizó hasta el 2003, donde se vuelve a incrementar para registrar en el 2008 un valor de 99,2 .

\section{Discusión}

\section{Configuración espacial y composición en el nivel de clase}

Los procesos de cambio en las cubiertas y uso del suelo, detectados en la cuenca del lago Cuitzeo correspondieron al aumento en el porcentaje de ocupación de los bosques cerrados y los matorrales durante los años de 1970 y 2000 y el decremento sustancial en dichas coberturas en 2008, lo que implicaría procesos de regeneración y transición forestal para los primeros años de este estudio y de deforestación para los últimos hasta el 2003, 
tal como lo reporta Mendoza et al. (2011). Otros cambios también fueron similarmente reportados en dicho estudio como el proceso de urbanización que en este estudio se revela con el aumento gradual de los asentamientos humanos a través del tiempo.

Una alta densidad de fragmentos de gran tamaño indica que los pastizales inducidos y cultivos presentan un alto grado de homogenización y dominancia, en todos los periodos de tiempo en comparación con las demás coberturas. Existen implicaciones ecológicas negativas causadas por el predominio de una cobertura introducida a través del tiempo $y$, por consiguiente la vegetación natural tiende a reducirse. Whittaker (1998) encontró que la modificación del paisaje forestal, en su mayoría por introducción de coberturas de pastos y cultivos, se asocia directamente con la extinción de la flora y fauna nativa, cuando estos dominan más del 30\% del área total del paisaje. Situaciones similares han sido reportadas en muchos más casos como en los estudios de Brook et al. (2003) y Brooks et al. (2002), en donde la extinción de la fauna se encuentra asociada a la pérdida de hábitat por remplazo de la vegetación natural. Asimismo, en el caso del centro de México, García-Romero (2002) identificó que procesos similares a este estudio, como el avance del frente urbano, la permanencia de las actividades agrícolas y la ganadería, han condicionado la invasión de especies exóticas y la expansión de otras especies naturales que toleran la incidencia de las actividades humanas.

Los matorrales registran tamaños de fragmentos relativamente pequeños y en general una alta densidad de fragmentos, indicando que su configuración espacial es heterogénea, característica de las coberturas fragmentadas. Sin embargo, de acuerdo con Tischendorf (2001) no siempre este comportamiento está relacionado con la fragmentación. En este sentido, la desaparición de fragmentos entre un periodo determinado puede afectar los valores del índice de PD y por ende el tamaño promedio de los fragmentos, indicando otro tipo de disturbio, como la deforestación, tal como puede ser en este caso si se trata de las coberturas arbóreas y arbustivas de la cuenca.

Es importante resaltar que en la tendencia temporal de los índices de MPS y PD de los bosques cerrados se pueden distinguir dos etapas particulares que pueden estar relacionadas con el estado de la conectividad estructural, la primera entre 1975 y 1996, cuando la densidad de fragmentos decrece y el tamaño promedio asciende, esto puede indicar un aumento en la conectividad estructural relacionado con el abandono de tierras de cultivo poco productivas y por programas de establecimiento forestal, eventos reportados por López et al. (2006). Por otro lado, dicho aumento, puede estar relacionado con el decrecimiento en el área de los bosques abiertos y semiabiertos entre 1975 y 1996, lo cual puede ser explicado por un proceso de densificación de los mismos, causando una conversión a bosques cerrados. En este sentido, los procesos de regeneración de las coberturas forestales incrementan la conectividad estructural en la región, como ha sido reportado en cuencas aledañas al área de estudio. Arredondo y García-Romero (2009), encontraron que los bosques maduros de la cuenca del río Tuxpan en Michoacán, se recuperaron a partir de vegetación secundaria durante 1975 y 2000 . El proceso de regeneración ha sido influenciado por la conversión de bosques abiertos y matorrales-pastizales a bosques cerrados de pino-encino.

La segunda etapa entre 1996 y 2008, por el contrario, puede indicar la existencia del proceso de fragmentación denominado "forest attrition" o desgaste del bosque (pérdida gradual de los fragmentos y áreas de bosque (Forman, 1995), el cual corresponde a una etapa "relictual" del estado de la cobertura boscosa (McIntyre \& Hobbs, 1999). Este proceso también fue observado para los matorrales entre 2003 y 2008.

La transformación de las coberturas, en este caso forestales, hacia áreas para pastoreo y cultivos, sobre todo en el periodo entre 2000 a 2008, generan graves impactos sobre la estructura de la vegetación natural de la cuenca ocasionando la degradación del hábitat para muchas especies, teniendo en cuenta que alrededor de ocho especies de plantas vasculares restringen su distribución a la cuenca y se reportan en serio peligro de extinción (Rzedowski, 2010 ), Por ejemplo el zapote prieto (Diospyros xolocotzii) que de acuerdo con Madrigal y Rzedowski (1988) es un árbol sumamente localizado y escaso, 
específicamente distribuido en una localidad cercana a Morelia donde solo se reportaron 20 individuos en una superficie de aproximadamente 10 hectáreas bajo la presión de la tala y la implantación de cultivos de maíz-frijol y calabaza. De acuerdo con Jones (2002) cuando las áreas bajo pastoreo se incrementan la probabilidad de extinción local de la vegetación nativa aumenta.

Galicia \& García-Romero (2007) reportan que los bosques templados del centro de México han sufrido una amplia transformación hacia áreas para pastoreo y cultivos entre 1970 y 2000, lo cual está relacionado con la tala ilegal y la extracción intensiva de resinas de las coníferas, que ha implicado una reducción sustancial en las coberturas forestales, caso contrario al de este estudio, en el que se reporta un aumento en el área de dichas coberturas entre 1975 y 2000, seguido de un decrecimiento considerable entre 2000 y 2008.

\section{Conectividad y aislamiento en el nivel de} clase

Los fragmentos de pastizales inducidos y cultivos se caracterizan por ser grandes y cercanos. En 1975 los fragmentos de dicha cobertura, en general, se encuentran menos aislados. Sin embargo, el notable decrecimiento en los valores del índice de proximidad hasta el 2003 indicando que los fragmentos se encuentran más aislados y presentan menor área. Esta tendencia puede estar dada por el proceso de matorralización y reforestación en este periodo de tiempo reportado por López et al. (2006). En este sentido, para 2008 se registró un incremento en las áreas de los fragmentos, así como en la conectividad estructural de los pastizales y cultivos.

Las coberturas de tipo arbóreo y arbustivo como los bosques cerrados, bosques abiertos y semiabiertos y los matorrales presentan en general registros muy bajos en el índice de conectancia, lo que indica que la conectividad estructural de las coberturas arbóreas y arbustivas en la cuenca del lago Cuitzeo es considerablemente baja sobre todo en las partes altas de la cuenca, donde todavía se conservan relictos de bosque nativo que registran una gran cantidad de especies de fauna.
Según Schondube et al. (2010), en el estudio de ecología espacial de las aves de la cuenca del lago Cuitzeo, se encontraron valores elevados de riqueza de especies, caso contrario en las áreas donde dominan las coberturas antrópicas como los pastos y cultivos. En este sentido, el aislamiento entre áreas que representan hábitats potenciales puede alterar la dispersión entre las áreas de hábitat óptimo.

De acuerdo con Mills y Allendorf (2006) principalmente la interrupción de la dispersión puede reducir el tamaño de las poblaciones afectando los patrones de flujo genético. Asimismo, Burkey \& Reed (2006), Pither y Taylor (1998) sugieren que el bajo grado de conectividad estructural entre fragmentos puede acarrear efectos sobre la ocupación de los fragmentos de hábitat sobre todo cuando se busca que haya recuperación de las poblaciones en áreas específicas.

De acuerdo con Mateucci (2004) los resultados obtenidos con el índice de cohesión son característicos de coberturas que presentan una alta representatividad en el total del paisaje como en el caso de las áreas agrícolas. Según Schumaker (1996), el índice de cohesión se incrementa a medida que la proporción de las clases también aumenta o viceversa. Por esta razón la tendencia de la cohesión es similar a la que se presenta en el porcentaje del índice PLAND. Estos resultados supondrían una alta agregación física entre los fragmentos de coberturas naturales, no obstante, si se compara con los resultados del índice de conectancia presentan muy poca relación y muy poca variabilidad, fluctuando entre 97.5 a 99.8, los cuales son similares a los reportados previamente (Schumaker, 1996; Saura \& Martínez-Millán, 2000; García-Gigorro \& Saura, 2005; Yu \& Nam, 2007; Mas et al., 2010; Mori \& Lertzman, 2011).

En este sentido, García-Gigorro \& Saura (2005) y Saura \& Pascual-Hortal (2007) reportan que el índice presenta un comportamiento inestable y registra valores altos (indicando alto grado de conectividad) en resoluciones espaciales finas al probarlo en mapas de coberturas boscosas con diferente tamaño de pixel. La anterior afirmación puede estar relacionada con los valores altos de cohesión registrados en el presente estudio; 
Asimismo, cuando el tamaño de pixel es relativamente pequeño $(<100 \times 100)$ se registran valores altos de conectividad que pueden ser falsos o ilegítimos, a medida que el tamaño de pixel aumenta el valor del índice de cohesión disminuye y se hace más sensible al aislamiento de los fragmentos (García-Gigorro \& Saura, 2005).

\section{Conclusiones}

El cálculo de métricas de paisaje aplicadas a una serie de datos de cobertura y uso del suelo resultó ser un método adecuado para evaluar las relaciones espaciales y la evolución temporal entre los tipos de cobertura y los fragmentos, dada la existencia de cinco puntos en el tiempo, lo cual permitió describir patrones, que de otra manera no se hubieran identificado.

El aporte de este estudio en términos de conservación es relevante en cuanto a que generó información acerca del estado estructural del paisaje y sus tendencias en el tiempo lo cual puede servir para la toma de decisiones concernientes al manejo integrado del paisaje en esta región.

Este método ofreció una base cuantitativa sólida para el seguimiento retrospectivo de la conectividad estructural. No obstante, corresponde a un enfoque exploratorio y descriptivo el cual puede complementarse con un análisis de conectividad funcional, lo cual integraría las relaciones espaciales en función de la capacidad de movilidad de las especies de fauna o de los flujos ecológicos en general aportando información adecuada para la identificación de áreas con potencial para la conservación.

\section{Referencias bibliográficas}

ARREDONDO-LEÓN, C. y GARCÍA-ROMERO, A. Uso de suelo y transformación del paisaje rural en el centro de México: el caso de la cuenca del río Tuxpan, Michoacán. Temas de Geografía Latinoamericana, 2009, p. 291-318.

BOTEQUILHA LEITAO, A.; MILLER, J.; AHERN, J. \& MCGARIGAL, K. Measuring Landscapes. A Planner's Handbook. Washington D.C.: Island press, 2006.
BROOK, B.W.; SODHI, N.S. \& NG, P.K. L. Catastrophic extinctions follow deforestation in Singapore. Nature, 2003, $N^{\circ} 424$, p. 420423.

BROOKS, T.; MITTERMEIER, G.; DA FONSECA, A.; RYLANDS, W.; KONSTANT, P.; FLICK, J.; PILGRIM, S.; OLDFIELD, G.; MARGIN, G. \& HILTON, C. Habitat loss and extinction in the hotspots of biodiversity. Conservation Biology, 2002, No 16, p. 909-923.

BURKEY, T.V. \& REED, D.H. The effects of habitat fragmentation on extinction risk: Mechanisms and synthesis. Songklanakarin Journal of Science and Technology, 2006, $\mathrm{N}^{\circ}$ 1, Vol. 28, p. 9-37.

CARLÓN ALLENDE, T. y MENDOZA, M.E. Análisis hidrometeorológico de las estaciones de la cuenca del lago de Cuitzeo. Investigaciones Geográficas (Mx), 2007, № 63, p. 56-76.

CORREA AYRAM, C.A.; MENDOZA, M.E.; PÉREZ SALICRUP, D.R. \& LÓPEZ GRANADOS, E. Identifying potential conservation areas in the Cuitzeo Lake basin, México by multitemporal analysis of landscape connectivity. Journal for nature conservation, 2014, Vol. 22, No 5, p. 424-453.

FORMAN, R.T.T. \& GODRON, M. Patches and Structural Components for a Landscape Ecology. BioScience, 1981, Vol. 31, № 10, p. 733-740.

FORMAN, R.T. Land mosaics: The ecology of landscapes and regions. New York: Cambridge University Press, 1995.

FRANKLIN, J. \& FORMAN, R.T. Creating landscape patterns by forest cutting: Ecological consequences and principles. Landscape Ecology, 1987, Vol. 1, № 1, p. 5-18.

GALICIA, L. \& GARCÍA-ROMERO, A. Land use and land cover change in highland temperate forests in the Izta-Popo National Park, central Mexico. Mountain Research and Development, 2007, Vol. 27, № 1, p.48-57.

GARCÍA-ROMERO, A. An evaluation of forest deterioration in the disturbed mountains of Western Mexico. Mountain Research and Development, 2002, Vol. 22, p. 270-277. 
GARCÍA-GIGORRO, S. \& SAURA, S. Forest fragmentation estimated from remotely sensed data: is comparison across scales possible? Forest Science, 2005, Vol. 51, N 1 , p. 51-63.

GERGEL, S.E. \& TURNER, M.G. Learning Landscape Ecology: A Practical Guide to Concepts and Techniques. New York: Springer, 2002.

GUSTAFSON, E.J. \& PARKER, G.R. Using an index of habitat patch proximity for landscape design. Landscape and Urban Planning, 1994, No29, p. 117-130.

GUSTAFSON, E.J. Quantifying landscape spatial pattern: what is the state of the art? Ecosystems, 1998, № 1, p. 143-156.

HARGIS, C.; BISSONETTE, J. \& DAVID, J. The behavior of landscape metrics commonly used in the study of habitat fragmentation. Landscape Ecology, 1998, Vol. 13, № 3, p. 167-186.

INSTITUTO NACIONAL DE ESTADÍSTICA, GEOGRAFÍA E INFORMÁTICA (INEGI). Censo de población y vivienda. México D.F.: Instituto Nacional de Estadística, Geografía e Informática, 1970.

INSTITUTO NACIONAL DE ESTADÍSTICA, GEOGRAFÍA E INFORMÁTICA (INEGI). Censo de población y vivienda. México D.F.: Instituto Nacional de Estadística, Geografía e Informática, 2010.

JONES, G. Impacts of grazing. Conservation Ecology, 2002, Vol. 6, №2. Disponible en Internet: http://www.consecol.org/vol6/ iss2/resp6/

KINDLMANN, P. \& BUREL, F. Connectivity measures: a review. Landscape Ecology, 2008, Vol. 23, № 8, p. 879-890.

LAITA, A.; KOTIAHO, J.S. \& MONKKONEN, M. Graph-theoretic connectivity measures: what do they tell us about connectivity? Landscape Ecology, 2011, Vol. 26, p. 951967.
LI, H. \& JIANGUO, W. Use and misuse of landscape indices. Landscape Ecology, 2004, Vol. 19, p. 389-399.

LÓPEZ-GRANADOS, E.; BOCCO, G.; MENDOZA, M.E.; VELÁZQUEZ, A. \& AGUIRRE, R. Peasant emigration and land-use change at the watershed level. A GIS-based approach in Central Mexico. Agricultural Systems, 2006, Vol. 90, p. 62-78.

MADRIGAL SÁNCHEZ, X. y RZEDOWS$\mathrm{KI}$, J. Una especie nueva de Diospyros (Ebenaceae) del municipio de Morelia, estado de Michoacán (México). Acta Botánica Mexicana, 1988, № 1, p. 3-6.

MAS, J.F.; GAO, Y. \& NAVARRETE, J.A. Sensitivity of landscape pattern metrics to classification approaches. Forest Ecology and Management, 2010, Vol. 259, № 7, p. 1215-1224.

MATEUCCI, S. Los índices de configuración del mosaico como herramienta para el estudio de las relaciones patrón-proceso. En: BUZAI, G. (compilador). Memorias del primer seminario argentino de geografía cuantitativa. Buenos Aires: I Seminario Argentino de Geografía Cuantitativa, 2004.

MCGARIGAL, K.; CUSHMAN, S.A.; NEEL, M.C. \& ENE, E. FRAGSTATS: Spatial Pattern Analysis Program for Categorical Maps. Amherst: University of Massachusetts, 2002. Disponible en Internet: http://www.umass. edu/landeco/research/fragstats/fragstats.html

MCINTYRE, S. \& HOBBS, R. A framework for conceptualizing human effects on landscapes and its relevance to management and research models. Conservation Biology, 1999, Vol. 13, N 6, p. 1282 - 1292.

MENDOZA, M.E.; BOCCO, G. y LÓPEZ, E. Regionalización ecológica. En: CONACYT. Regionalización ecológica, conservación de recursos naturales y ordenamiento territorial en la cuenca del lago de Cuitzeo, Michoacán. México D.F.: CONACYT, 2001.

MENDOZA M.E.; LÓPEZ, E. y PÉREZ -SALICRUP, D. R. Evaluación espacial y multitemporal de los cambios de cobertura y uso del suelo en la cuenca del lago de Cuitzeo: implicaciones para la sucesión forestal y el 
mantenimiento de la diversidad vegetal. México D.F.: Universidad Nacional Autónoma de México, 2010.

MENDOZA, M.E.; LÓPEZ GRANADOS, E.; GENELETTI, D.; PÉREZ-SALICRUP, D.R. \& SALINAS, V. Analyzing land cover and land use change processes at watershed level: A multitemporal study in the Lake Cuitzeo Watershed, Mexico (1975-2003). Applied Geography, 2011, №31, p. 237-250.

MILLS, L.S. \& ALLENDORF, F.W. The onemigrant-per-generation rule in conservation and management. Conservation Biology, 1996, No 10, p. 1509-1518.

MORALES, L.M. Características físicas de la Cuenca del lago Cuitzeo: Área de estudio. En: CRAM, S.; GALICIA, L. y ALCÁNTARA, I. I. (compiladores). Atlas de la cuenca del lago de Cuitzeo: un análisis de la geografía y su entorno socioambiental. México D.F.: UNAMUMSNH, 2010, p. 20-24.

MORI, A.S. \& LERTZMAN, K.P. Historic variability in fire-generated landscape heterogeneity of subalpine forests in the Canadian Rockies. Journal of Vegetation Science, 2011, $\mathrm{N}^{\circ} 22$, p. $45-58$.

NEEL, M.C.; CUSHMAN, S.A. \& MCGARIGAL, K. Behavior and stability of landscape metrics across controlled gradients of landscape structure. Landscape Ecology, 2004, N 19 , p. $455-534$.

O'NEILL, R.V.; KRUMMEL, J.R.; GARDNER, R.H.; SUGIHARA, G.; JACKSON, B.; DEANGELIS, D.L.; MILNE, B.T.; TURNER, M.G.; ZYGMUNT, B.; CHRISTENSEN, S.W.; DALE, V.H. \& GRAHAM, R.L. Indices of landscape pattern. Landscape Ecology, 1988, Vol. I, No3, p. 153-162.

PRIEGO, A.; BOCCO, G.; MENDOZA, M. y GARRIDO, A. Propuesta para la generación semiautomatizada de unidades de paisaje. México D.F.: Serie Planeación Territorial, 2010.

PITHER, J. \& TAYLOR, P.D. An experimental assessment of landscape connectivity. Oikos, 1998, № 83, p. 166-174.
RITTERS, K.H.; O'NEILL, R.V.; HUNSAKER, C.T.; WICKHAM, J.D.; YANKEE, D.H.; TIMMINS, S.P.; JONES, K.B. \& JACKSON, B.L. A factor analysis of landscape pattern and structure metrics. Landscape Ecology, 1995, Vol. 10, $\mathrm{N}^{\circ} 1$, p. 23-39.

SARMIENTO, F.O. Diccionario de ECología de Paisajes, Conservación y Desarrollo Sustentable para Latinoamérica. Quito: Editorial Abya-Yala, 2001.

RZEDOWSKI, J. Flora de la cuenca del lago Cuitzeo. En: CRAM, S.; GALICIA, L. y ALCÁNTARA, I. (compiladores). Atlas de la Cuenca del lago Cuitzeo: Análisis de su geografía y entorno socioambiental. México D.F.: UNAM-UMSHN, 2010, p. 102-107.

SAURA, S. \& MARTÍNEZ-MILLÁN, J. Landscape patterns simulation with a modified random clusters method. Landscape ECOlogy, 2000, Vol. 15, № 7, p. 661-678.

SAURA, S. \& MARTÍNEZ-MILLÁN, J. Sensitivity of Landscape Pattern Metrics to Map Spatial Extent. Photogrammetric Engineering \& Remote Sensing, 2001, Vol. 67, No 9, p. 1027-1036.

SAURA, S. \& PASCUAL-HORTAL, L. A new habitat availability index to integrate connectivity in landscape conservation planning: comparison with existing indices and application to a case study. Landscape and Urban Planning, 2007, Vol. 83, $\mathrm{N}^{\circ} 2-3$, p. 91-103.

SCHONDUBE, J.E.; MACGREGOR-FORS, I.; MORALES-PÉREZ, L.; MENDOZA, M. y LÓPEZ, E. Ecología espacial de aves en la Cuenca de Cuitzeo. En: CRAM, S.; GALICIA, L. y ALCÁNTARA, I. (compiladores). Atlas de la Cuenca de lago Cuitzeo: Análisis de su geografía y entorno socioambiental. México D.F.: UNAM-UMSHN, 2010, p. 102-107.

SCHUMAKER, N.H. Using landscape indices to predict habitat connectivity. Ecology, 1996, No 77 , p. 1210-1225.

TAYLOR, P.D.; FAHRIG, L. \& WITH, K. Landscape connectivity: A return to basics. In: CROOKS, K.R. \& SANJAYAN, M. (editors). 
Connectivity Conservation. Cambridge: Cambridge University Press, 2006, p. 29-43.

TISCHENDORF, L. Can landscape indices predict ecological processes consistently? Landscape Ecology, 2001, Vol. 16, №3, p. 235-254.

TURNER, M. G. Spatial and temporal analysis of landscape pattern. Landscape ECOlogy, 1990, $\mathrm{N}^{\circ} 4$, p. 21-30.

VIDAL ZEPEDA, R. Características físicas de la cuenca del lago Cuitzeo: Clima. En: CRAM, S.; GALICIA, L. y ALCÁNTARA, I. (compiladores). Atlas de la Cuenca de lago Cuitzeo: Análisis de su geografía y entorno socioambiental. México D.F.: UNAM-UMSHN, 2010, p. 24-28.

WITH, K.A. \& KING, A.W. Dispersal success on fractal landscapes: a consequence of lacunarity thresholds. Landscape Ecology, 1999, No 14, p. 73.82
WHITTAKER, R.J. Island Biogeography: ecology, evolution, and conservation. Oxford: Oxford University Press, 1998.

YU, X.J. \& NG, C.N. Spatial and temporal dynamics of urban sprawl along two urbanrural transects: A case study of Guangzhou, China. Landscape and Urban Planning, 2007, Vol. 79, No 1, p. 96-109.

ZARAGOZÍ, B.; BELDA, A.; LINARES, J.; MARTÍNEZ-PÉREZ, J.E.; NAVARRO, J.T. \& ESPARZA, J. A free and open source programming library for landscape metrics calculations. Environmental Modelling \& Software, 2012, Vol. 31, p. 131-140. 
